\title{
Higgs inflation with non-minimal derivative coupling to gravity
}

\author{
L. N. Granda* D.F. Jimenez ${ }^{\dagger}$ W. Cardona ${ }^{\ddagger}$ \\ Departamento de Fisica, Universidad del Valle \\ A.A. 25360, Cali, Colombia
}

\begin{abstract}
We consider an extension of Higgs inflation in which the Higgs field is nonminimally coupled to gravity through its kinetic term. We analyzed power-law coupling functions with positive or negative integer power and found that the Higgs boson can drive a successful inflation only for the cases $n=2,1,0,-1$. Theoretical predictions for both tensor to scalar ratio $r$ and scalar spectral index $n_{s}$ are within the 2018 Planck 95\% CL. The behavior of the self coupling $\lambda$ with respect to the scalar field at the horizon crossing was obtained, and It was found that it can take values in the interval $\lambda \sim\left(10^{-7}, 0.3\right)$.
\end{abstract}

\section{Introduction}

Several shortcomings (e.g., flatness, horizon, unobserved heavy magnetic monopoles [see for instance [1, 2, 3, 4, 5, 6]]) pervaded the standard hot big-bang scenario until around 1980 when the inflationary paradigm started to be constructed. Then, cosmologists initiated to find out that an epoch of very rapid expansion in the early

*luis.granda@correounivalle.edu.co

†jimenez.diego@correounivalle.edu.co

${ }^{\ddagger}$ wilmar.cardona@correounivalle.edu.co 
universe could lead to a possible, plausible solution of the standard hot big-bang problems [7, 8, 9, 10, 11, 12].

Among the wide variety of inflationary models, the simplest realization of inflation occurs with a minimally coupled scalar field [13, 14]. While the scalar field potential dominates the energy density of the universe, there is a period of nearly exponential growth and the drawbacks of the standard hot big-bang scenario get simply solved. Moreover, inflation also provides an explanation for the observed structures in the Universe [15, 16, 17, 18, 19, 20]. Quantum fluctuations in the scalar field would have led to small inhomogeneities in the energy density that later became the structures we observe, namely, Cosmic Microwave Background (CMB) anisotropies and inhomogeneities in the mass distribution.

A number of approaches differing from canonical inflation have been investigated over the past years. An early inflationary regime is found in models such as nonminimally coupled scalar fields [21, 22, 23], kinetic inflation [24], $\alpha$ attractor models [25, 26, 27, 28], Dirac-Born-Infeld (DBI) inflation [29, 30, 31, 32], string theory inspired inflation [33, 34, 35], vector inflation [36, 37, 38], inflaton potential in supergravity [39, 40, 41, and Galileon models [42, 43, 44, 45, 46, 47, 48, 49], also including scalar fields with non-minimal derivative coupling to the Einstein tensor [50, 51, 52, 53, 54, 55]. Scalar fields are abundant in fundamental theories of matter beyond the standard model of particle physics. Nevertheless, the only known fundamental scalar quantum field is the Higgs field discovered by the CERN collaborations ATLAS and CMS [56, 57]. Over the past years, intense research has been carried out trying to identify the Higgs scalar with the field causing the early inflationary period. Although this identification could in principle be possible, it has proven to be non-trivial [58]. The limitations of the semiclassical approach to slow-roll inflation with the Higgs field non-minimally coupled to gravity have been pointed out in [59, 60, 61, 62, 63], where the issue of unitarity bound has been addressed. The study of the unitarity issue in the new Higgs inflation with non-minimal kinetic coupling to the Einstein tensor was performed in [64, 65, 66, 63], where it was shown that in this scenario there is not perturbative unitarity violation.

An interesting framework for inflation is provided by theories including curvature 
corrections such as

$$
\frac{\beta}{\phi^{n}} G_{\mu \nu} \partial^{\mu} \phi \partial^{\nu} \phi
$$

This sort of theories add gravitational friction and make the scalar field to roll slower compared to the canonical scalar field. Moreover, this sort of theories are embedded in the most general Lagrangian giving second order equations, the so-called Horndeski Lagrangian. There are not strongly proved physical justifications to consider higher derivative terms in the action of the scalar field to study the inflationary phenomena but, among few theoretical motivations, we can mention the fact that the type of couplings we are considering here lead to second-order field equations, avoiding the appearance of Ostrogradsky instabilities and leading to ghost-free theory, providing more general second order Lagrangian formalism. Terms of this type, with field-dependent couplings, also appear in low energy effective action of string theory [67, 68]. It has been shown also that couplings such as Gauss-Bonnet provide the possibility of avoiding the initial singularity [69, 70]. An appealing motivation to study such corrections to canonical scalar field is that at the high-curvature regime typical of inflation, these corrections could become appreciable and affect the outcome of inflation. In the case of the Higgs potential, for instance, one of the effects of the higher derivative terms is the reduction of the self coupling of the Higgs boson, so that the spectra of primordial density perturbations are consistent with the present observational data [71, 72]

The main focus in this paper will be to answer to what extent the Higgs field only including a non-minimal kinetic coupling to gravity can successfully render an inflationary regime in the early universe. In section 2 we explain the model and provide the theoretical framework for our study. Then we show in section 3 that this model is compatible with current Planck constraints on the scalar spectral index $n_{s}$ and the tensor to scalar ratio $r$. Concluding remarks are presented in section 4 . 


\section{Theoretical framework}

We consider a generalization of the standard Higgs action where the Higgs field $\phi$ and its derivatives are coupled to the gravity, such that

$$
S=\int d^{4} x \sqrt{-g}\left[\frac{R}{2 \kappa^{2}}-\frac{1}{2} \partial_{\mu} \phi \partial^{\mu} \phi-\frac{\lambda}{4} \phi^{4}+\frac{\beta}{\phi^{n}} G_{\mu \nu} \partial^{\mu} \phi \partial^{\nu} \phi\right],
$$

where $R$ is the Ricci scalar, $\kappa^{2} \equiv 8 \pi G_{N}$ with $G_{N}$ the bare Newton's constant, $G_{\mu \nu}$ is the Einstein tensor, $\beta$ is an arbitrary parameter with dimension of $(\text { mass })^{n-2}$ and $n$ is an integer. We will further assume that during inflation one can neglect the vacuum expectation value of the Higgs scalar so that a quartic potential is a good approximation for the Higgs potential. The self-coupling constant $\lambda$ is fixed experimentally by the LHC results, which impose the constrain $\lambda \simeq 0.13$ at the electroweak scale [73].

In the flat Friedmann-Lemaitre-Robertson-Walker (FLRW) metric the equations of motion take the form

$$
\begin{gathered}
H^{2}=\frac{\kappa^{2}}{3}\left(\frac{1}{2} \dot{\phi}^{2}+\frac{\lambda}{4} \phi^{4}+9 \frac{\beta}{\phi^{2}} H^{2} \dot{\phi}^{2}\right) \\
2 \dot{H} F\left(1-\frac{\kappa^{2} \beta \dot{\phi}^{2}}{\phi^{n}}\right)=-\kappa^{2}\left(\dot{\phi}^{2}+6 \beta \frac{H^{2} \dot{\phi}^{2}}{\phi^{n}}-4 \beta \frac{H \dot{\phi} \ddot{\phi}}{\phi^{n}}+2 n \beta \frac{H \dot{\phi}^{3}}{\phi^{n+1}}\right)
\end{gathered}
$$

and

$$
\ddot{\phi}+3 H \dot{\phi}+\lambda \phi^{3}+6 \beta H\left(3 H^{2}+2 \dot{H}\right) \frac{\dot{\phi}}{\phi^{2}}+6 \beta H^{2} \frac{\ddot{\phi}}{\phi^{2}}-6 \beta H^{2} \frac{\dot{\phi}^{2}}{\phi^{3}}=0 .
$$

Next we define the slow-roll parameters, which involve the interaction terms in the model (2.1), as (see [74])

$$
\begin{gathered}
\epsilon_{0}=-\frac{\dot{H}}{H^{2}}, \quad \epsilon_{1}=\frac{\dot{\epsilon}_{0}}{H \epsilon_{0}} \\
k_{0}=3 \beta \kappa^{2} \frac{\dot{\phi}^{2}}{\phi^{n}}, \quad k_{1}=\frac{\dot{k}_{0}}{H k_{0}}
\end{gathered}
$$

Note that for the simple canonical scalar field, $\frac{1}{2} \dot{\phi}^{2}+V(\phi)$, the above $H$-defined slow-roll parameters are related to the standard $V$-defined slow-roll parameters

$$
\epsilon_{v}=\frac{M_{p}^{2}}{2}\left(\frac{V^{\prime}}{V}\right)^{2}, \quad \eta_{v}=M_{p}^{2} \frac{V^{\prime \prime}}{V}
$$


in the way

$$
\epsilon_{0}=\epsilon_{v}, \quad \text { and } \quad \epsilon_{1}=4 \epsilon_{v}-2 \eta_{v}
$$

where the slow-roll approximation

$$
2 H^{2} \simeq \kappa^{2} V, \quad 3 H \dot{\phi}+V^{\prime} \simeq 0
$$

was used.

Writing the cosmological equations 2.2 - 2.4 in terms of the slow-roll, parameters (2.5), (2.6), we can express the derivative of the scalar field and the potential as

$$
\begin{gathered}
V=M_{p}^{2} H^{2}\left[3-\epsilon_{0}-2 k_{0}-\frac{1}{3} k_{0}\left(k_{1}-\epsilon_{0}\right)\right] \\
\dot{\phi}^{2}=M_{p}^{2} H^{2}\left[2 \epsilon_{0}-2 k_{0}+\frac{2}{3} k_{0}\left(k_{1}-\epsilon_{0}\right)\right]
\end{gathered}
$$

from which it becomes clear that $\dot{\phi}^{2}<<V$ under the slow-roll conditions $\epsilon_{0}, k_{0}, \ldots<<$ 1. The slow-roll conditions $\dot{\phi}^{2}<<V=\frac{\lambda}{4} \phi^{4}, \ddot{\phi}<<3 H \dot{\phi}, k_{0}, k_{1}<<1$ allow to write the Eqs. (2.2)- 2.4 in the following approximate way

$$
\begin{gathered}
H^{2} \simeq \frac{\kappa^{2}}{3} V=\frac{\kappa^{2}}{3}\left(\frac{\lambda}{4} \phi^{4}\right) \\
\dot{H} \simeq-\frac{\kappa^{2}}{2}\left(\dot{\phi}^{2}+6 \beta \frac{H^{2} \dot{\phi}^{2}}{\phi^{n}}\right) \\
3 H \dot{\phi}+V^{\prime}+18 \beta \frac{H^{3} \dot{\phi}}{\phi^{n}} \simeq 0
\end{gathered}
$$

From these equations we can see that the potential term gives the dominant contribution to the Hubble parameter, and Eqs. 2.12, (2.13) determine the role of the kinetic coupling in the slow-roll dynamics. These equations allow to find the number of $e$-folds as

$N=\int_{\phi_{I}}^{\phi_{E}} \frac{H}{\dot{\phi}} d \phi=-\int_{\phi_{i}}^{\phi_{\text {end }}} \frac{3 H^{2}}{V^{\prime}}\left(1+6 \beta \frac{H^{2}}{\phi^{n}}\right) d \phi=-\int_{\phi_{i}}^{\phi_{\text {end }}} \frac{V}{M_{p}^{2} V^{\prime}}\left(1+2 \beta \frac{V}{M_{p}^{2} \phi^{n}}\right) d \phi$

where $\phi_{i}$ and $\phi_{\text {end }}$ are the values of the scalar field at the beginning and end of inflation respectively. The scalar field at the end of inflation can be found from the condition 
$\epsilon_{0}\left(\phi_{\text {end }}\right)=1$, and $\phi_{i}$, which is the scalar field at the horizon crossing, is calculated from (2.14) by giving the appropriate number of e-folds such that the inflationary observables take values consistent with current observations. In our case we observed that given $N \simeq 60$ produce the necessary inflation to set the values of the scalar spectral index and the tensor-to-scalar ratio in the most appropriate regions, within the capabilities of the model. For the $\phi^{4}$ potential we find

$$
N \approx \int_{\phi_{i}}^{\phi_{\text {end }}} \frac{1}{4} \phi\left(\frac{1}{2} \beta \lambda \phi^{4-n}+1\right) d \phi,
$$

In order to obtain the inflationary observable magnitudes we need to calculate the power spectrum of the scalar and tensor perturbations, which was presented for a general class scalar-tensor models in [74]. For our particular case, as follows from [74], the scalar spectral index and the tensor-to-scalar ratio are given by (in first order in slow-roll parameters)

$$
n_{s}=1-2 \epsilon_{0}-\epsilon_{1}, \quad r=16 \epsilon_{0}
$$

where we can see that the non-minimal kinetic coupling to curvature does not affect the standard consistency relation, at least up to first order in slow-roll parameters. These magnitudes can be written as functions of the scalar field and are evaluated at the horizon crossing. To this end we express the Hubble parameter and its derivatives, $\dot{H}$ and $\ddot{H}$, together with the derivatives of the scalar field $\dot{\phi}, \ddot{\phi}$ in terms of the potential and the kinetic coupling function, reducing all magnitudes to functions of the scalar field. We can illustrate this procedure for the general case of model with potential $V(\phi)$ and kinetic coupling $F_{1}(\phi)$, writing the Eqs. 2.11)-2.13) in the form

$$
\begin{gathered}
H^{2} \simeq \frac{\kappa^{2}}{3} V(\phi) \\
\dot{H} \simeq-\frac{\kappa^{2}}{2}\left(\dot{\phi}^{2}+6 H^{2} F_{1}(\phi) \dot{\phi}^{2}\right) \\
3 H \dot{\phi}+V^{\prime}+18 H^{3} F_{1}(\phi) \dot{\phi} \simeq 0 .
\end{gathered}
$$

The Eq. 2.17) gives directly $H(\phi)$, while combining the three equations and taking the necessary derivatives we find

$$
\dot{H}=-\frac{V^{\prime}(\phi)^{2}}{6 V(\phi)\left(1+2 \kappa^{2} F_{1}(\phi) V(\phi)\right)}
$$




$$
\begin{gathered}
\dot{\phi}=\frac{V^{\prime}(\phi)}{\sqrt{3} \kappa \sqrt{V}\left(1+2 \kappa^{2} F_{1}(\phi) V(\phi)\right)} \\
\ddot{H}=\frac{V^{\prime}(\phi)^{2}\left[V^{\prime}(\phi)^{2}+V\left(4 \kappa^{2} F_{1}(\phi) V^{\prime}(\phi)^{2}-2 V^{\prime \prime}(\phi)\right)+2 \kappa^{2} V(\phi)^{2}\left(F_{1}^{\prime}(\phi) V^{\prime}(\phi)-2 F_{1}(\phi) V^{\prime \prime}(\phi)\right)\right]}{6 \sqrt{3} \kappa V(\phi)^{5 / 2}\left(1+2 \kappa^{2} F_{1}(\phi) V(\phi)\right)^{3}} \\
\ddot{\phi}=-\frac{V^{\prime}(\phi)\left[V^{\prime}(\phi)^{2}+V(\phi)\left(6 \kappa^{2} F_{1}(\phi) V^{\prime}(\phi)^{2}-2 V^{\prime \prime}(\phi)\right)+4 \kappa^{2} V(\phi)^{2}\left(F_{1}^{\prime}(\phi) V^{\prime}(\phi)-F_{1}(\phi) V^{\prime \prime}(\phi)\right)\right]}{6 \kappa^{2} V(\phi)^{2}\left(1+2 \kappa^{2} F_{1}(\phi) V(\phi)\right)^{3}}
\end{gathered}
$$

Replacing these results in (2.5) and (2.6), and turning to the specific case $V=\lambda \phi^{4} / 4$ and $F_{1}=\beta / \phi^{n}$, after some algebra we find

$$
\begin{gathered}
\epsilon_{0}=\frac{16}{2 \phi^{2}+\alpha \phi^{6-n}}, \quad \epsilon_{1}=\frac{\left[32 \phi^{n}+8(6-n) \alpha \phi^{4}\right] \phi^{n-2}}{\left(2 \phi^{n}+\alpha \phi^{4}\right)^{2}} \\
k_{0}=\frac{16 \alpha \phi^{n+2}}{\left(2 \phi^{n}+\alpha \phi^{4}\right)^{2}}, \quad k_{1}=\frac{\left[16(n-2) \phi^{n}+8(6-n) \alpha \phi^{4}\right] \phi^{n-2}}{\left(2 \phi^{n}+\alpha \phi^{4}\right)^{2}}
\end{gathered}
$$

where

$$
\alpha \equiv \beta \lambda
$$

and we have set $M_{p}=1$. Finally, using (2.16), we can write the scalar spectral index and the tensor-to-scalar ratio in terms of the scalar field for the model (2.1) as

$$
\begin{gathered}
n_{s}=\left.\frac{\alpha^{2} \phi^{10}+4 \alpha\left(2 n+\phi^{2}-20\right) \phi^{n+4}+4\left(\phi^{2}-24\right) \phi^{2 n}}{\phi^{2}\left(\alpha \phi^{4}+2 \phi^{n}\right)^{2}}\right|_{\phi=\phi_{i}}, \\
r=\left.\frac{256}{2 \phi^{2}+\alpha \phi^{6-n}}\right|_{\phi=\phi_{i}} .
\end{gathered}
$$

In order to analyze the observational constraints on the model, and particularly, how they influence the self coupling $\lambda$, we resume here some aspects of the second order formalism (see [74] for details).

For the scalar and tensor perturbations we can write the power spectra respectively as

$$
P_{\xi}=A_{S} \frac{H^{2}}{(2 \pi)^{2}} \frac{\mathcal{G}_{S}^{1 / 2}}{\mathcal{F}_{S}^{3 / 2}}, \quad P_{T}=16 A_{T} \frac{H^{2}}{(2 \pi)^{2}} \frac{\mathcal{G}_{T}^{1 / 2}}{\mathcal{F}_{T}^{3 / 2}}
$$

where

$$
\mathcal{F}_{S}=c_{S}^{2} \mathcal{G}_{S}, \quad \mathcal{F}_{T}=c_{T}^{2} \mathcal{G}_{T}
$$


and

$$
A_{S}=\frac{1}{2} 2^{2 \mu_{s}-3}\left|\frac{\Gamma\left(\mu_{s}\right)}{\Gamma(3 / 2)}\right|^{2}, \quad A_{T}=\frac{1}{2} 2^{2 \mu_{T}-3}\left|\frac{\Gamma\left(\mu_{T}\right)}{\Gamma(3 / 2)}\right|^{2},
$$

where in terms of the slow-roll parameters, up to first order

$$
\mathcal{G}_{S}=M_{p}^{2} \epsilon_{0}
$$

and

$$
\begin{gathered}
c_{S}^{2}=1 \\
\mathcal{G}_{T}=M_{p}^{2}\left(1-\frac{1}{3} k_{0}\right)
\end{gathered}
$$

and

$$
c_{T}^{2}=\frac{3+k_{0}}{3-k_{0}}
$$

where $c_{S}$ and $c_{T}$ are the velocities of scalar and tensor perturbations respectively, and all magnitudes are evaluated at the moment of horizon exit when $c_{S} k=a H$.

On the other hand, the relative contribution to the power spectra of tensor and scalar perturbations, defined as the tensor/scalar ratio $r$, given by

$$
r=\frac{P_{T}(k)}{P_{\xi}(k)}
$$

can be written as

$$
r=16 \frac{\mathcal{G}_{T}^{1 / 2} \mathcal{F}_{S}^{3 / 2}}{\mathcal{G}_{S}^{1 / 2} \mathcal{F}_{T}^{3 / 2}}=16 \frac{\mathcal{G}_{S}}{\mathcal{G}_{T}}
$$

where we used fact that $A_{T} / A_{S} \simeq 1$ and $c_{T} \simeq 1$ when evaluated at the limit $\epsilon_{0}, \ldots<<$ 1. Using 2.32) and 2.34), gives the consistency relation for $r$ 2.16). The equation 2.29) for $P_{\xi}$ with 2.31 for $A$ allows to find scale of the Hubble parameter during inflation by using the COBE normalization for the power spectra, which can be written as 2.29

$$
P_{\xi}=A_{S} \frac{H^{2}}{(2 \pi)^{2}} \frac{\mathcal{G}_{S}^{1 / 2}}{\mathcal{F}_{S}^{3 / 2}} \sim \frac{H^{2}}{2(2 \pi)^{2}} \frac{1}{\mathcal{F}_{S}} \sim \frac{H^{2}}{(2 \pi)^{2} M_{p}^{2}} \frac{1}{2 \epsilon_{0}}
$$

where at the limit $\left(\epsilon_{0}, \epsilon_{1}, \ldots\right) \rightarrow 0$ we used the approximation $A_{S} \rightarrow 1 / 2$. According to COBE normalization $P_{\xi} \simeq 2.4 \times 10^{-9}$. Then,

$$
H^{2} \sim 2\left(2.4 \times 10^{-9}\right)(2 \pi)^{2} \epsilon_{0} .
$$


On the other hand, from the tensor/scalar ratio we find

$$
P_{T}=r P_{\xi} \sim \frac{2 V}{3 \pi^{2} M_{p}^{4}} \quad \Rightarrow \quad V \sim\left(2.4 \times 10^{-9}\right) \frac{3 \pi^{2}}{2} r M_{p}^{4},
$$

which also follows from (2.39). For the Higgs potential this last result allows to find the self coupling $\lambda$ in terms of the scalar field at the beginning of inflation as

$$
\left.\lambda \sim \frac{1536\left(2.4 \times 10^{-9}\right) \pi^{2}}{\phi^{4}\left(2 \phi^{2}+\alpha \phi^{6-n}\right)}\right|_{\phi_{i}}
$$

From this result we can see that $\lambda$ decreases with the increment of the scalar field at the horizon crossing. As will be shown in some numerical cases, large field inflation can give $\lambda \sim 10^{-6}$. When $\phi_{i}$ takes almost constant small values, at large $\alpha$, the self coupling can take values of the order $\lambda \sim 0.1$. In the next section we consider some numerical cases.

\section{Results}

In this section we show theoretical predictions of the model for both tensor to scalar ratio $r$ and scalar spectral index $n_{s}$. In Fig. 1 we consider four coupling functions corresponding to $n=-1,0,1,2$ and show the behavior of $r$ and $n_{s}$ for a wide $\alpha$ interval. Since the field at horizon crossing depends on $\alpha$, it is therefore important to make sure that the inflationary period occurs for field values such that the current restrictions on the self coupling $\lambda$ of the Higgs boson are satisfied. In Fig. 2 we show the behavior of the inflaton field at the horizon crossing,60 e-folds before the end of inflation, with respect to $\alpha$. The left graphic shows that in the region $\alpha \lesssim 10^{3}$, the scalar filed takes values $\phi_{i} \gtrsim M_{p}$ leading to large field inflation, and the right graphic shows that in the region $\alpha \sim 10^{12}-10^{14}$ the scalar field takes values of the order $\phi_{i} \sim 10^{-2}$. This affects the behavior of the self coupling $\lambda$ as shown in Figs. 3 an 4 below, where $\lambda$ can take values in the interval $10^{-7} \lesssim \lambda \lesssim 0.3$, covering a wide spectrum of possible values of the self coupling, according to the different Higgs boson phenomenological restrictions. In Fig. 2 we show the variation of the scalar field at horizon crossing. Notice that at large values $\left(\alpha \sim 10^{13}-10^{14}\right)$ the field $\phi_{i} \sim 10^{-2} M_{p}$ 


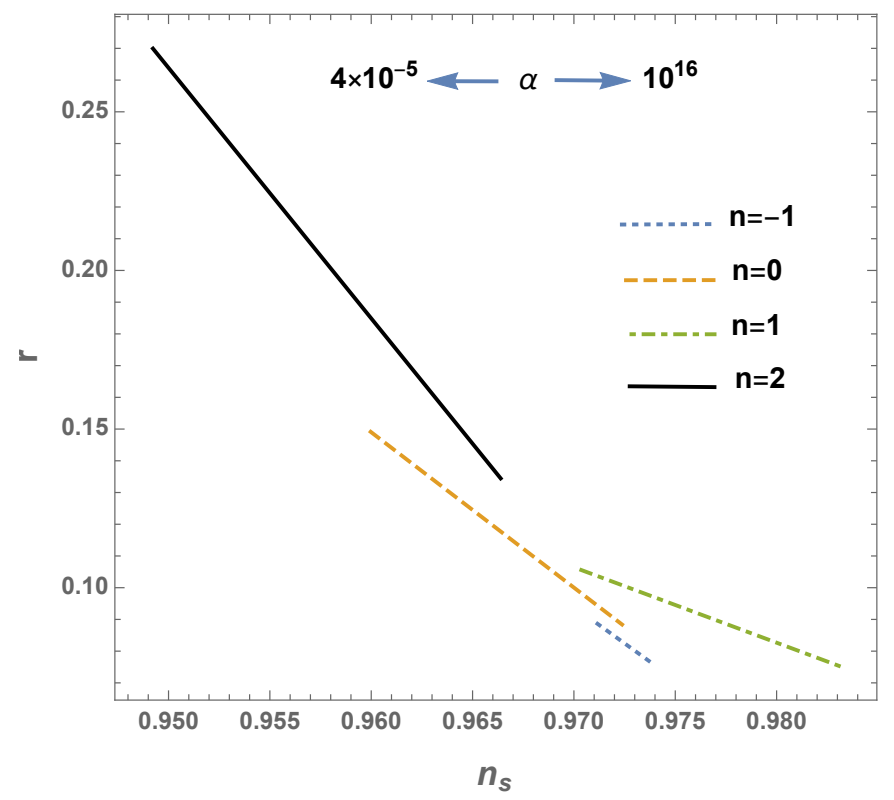

Figure 1: Parametric plot of $r$ vs $n_{S}$ for $4 \times 10^{-5}<\alpha<10^{16}$ with $N=60$, for coupling functions corresponding to $n=-1,0,1,2$. For the models $n=-1$ and $n=0$ the inflationary observables $n_{s}$ and $r$ are more close to the region favored by current observational data. 

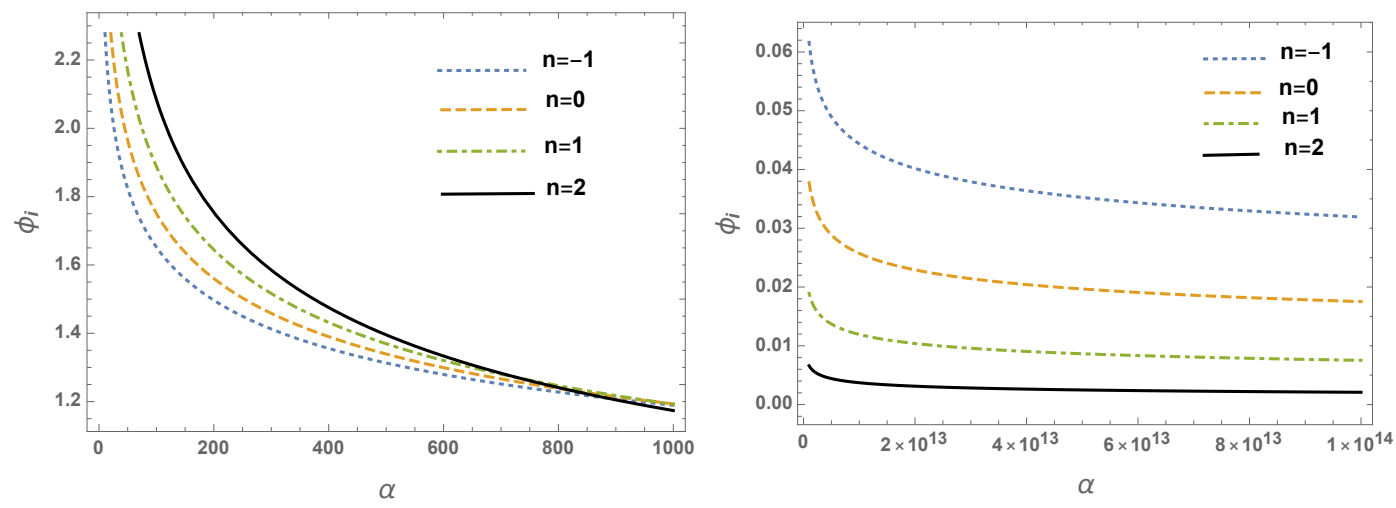

Figure 2: The inflaton at the horizon crossing as function of the parameter $\alpha$ for $n=-1,0,1,2$ with $N=60$. The left graphic shows large field inflation scenario where, for $\alpha \lesssim 10^{3}$, the scalar filed takes values $\phi_{1} \gtrsim M_{p}$. The right graphic shows the small field inflation, where starting from $\alpha \gtrsim 10^{13}$, the initial scalar field becomes $\phi_{i} \lesssim 10^{-2} 60$-e-folds before the end of inflation and continue decreasing with the increase of $\alpha$.

and at small $\alpha, \alpha \lesssim 10^{3}$ one finds $\phi_{1} \gtrsim M_{p}$.

In Figs. 3, 4 we show the behavior of the self coupling $\lambda$ with respect to the scalar field at the horizon crossing, where due to the difference in the scale we show two different intervals for $\phi_{i}$. It is important to note that along both $\alpha$-intervals, which results in the corresponding intervals for the scalar field in Figs. 3 and 4, the scalar spectral index $n_{s}$ and tensor/scalar ratio $r$ remain practically unchanged for each case, taking the following values

$$
\begin{aligned}
& n=-1 \quad \Rightarrow \quad n_{s} \simeq 0.974, r \simeq 0.075 \\
& n=0 \quad \Rightarrow \quad n_{s} \simeq 0.972, r \simeq 0.088 \\
& n=1 \quad \Rightarrow \quad n_{s} \simeq 0.970, r \simeq 0.106 \\
& n=2 \quad \Rightarrow \quad n_{s} \simeq 0.966, r \simeq 0.134
\end{aligned}
$$

the difference between the values at $\alpha \sim 10^{3}$ and $\alpha \sim 10^{16}$ is in the order of $10^{-4}$ or less, while changes a little more for $\alpha \lesssim 10^{-3}$ where the difference can be of the order of $10^{-2}-10^{-1}$ (which is more accentuated for $r$ ), getting closer to the weak coupling 


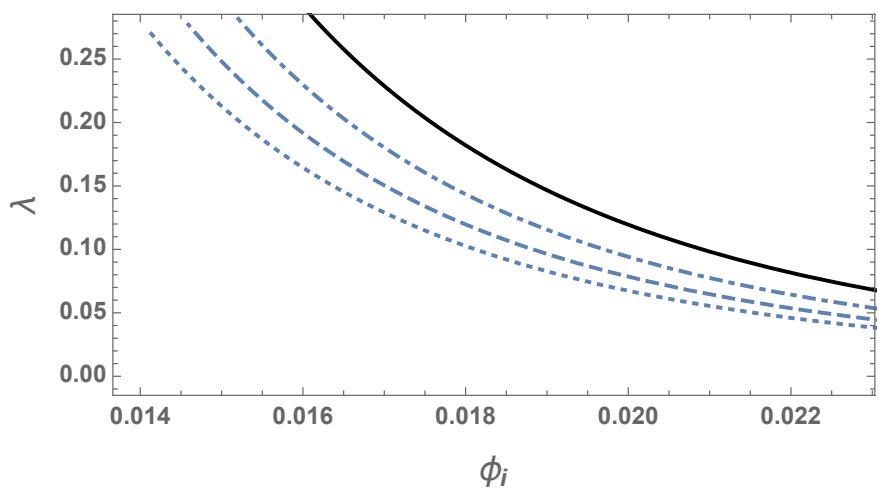

Figure 3: The behavior of the self coupling $\lambda$ with respect to the scalar field at the horizon crossing, $60 e$-folds before the end of inflation, for the four models corresponding to $n=-1,0,1,2$. These curves correspond to the small-field inflation, giving $\lambda \sim 10^{-2}-0.28$.

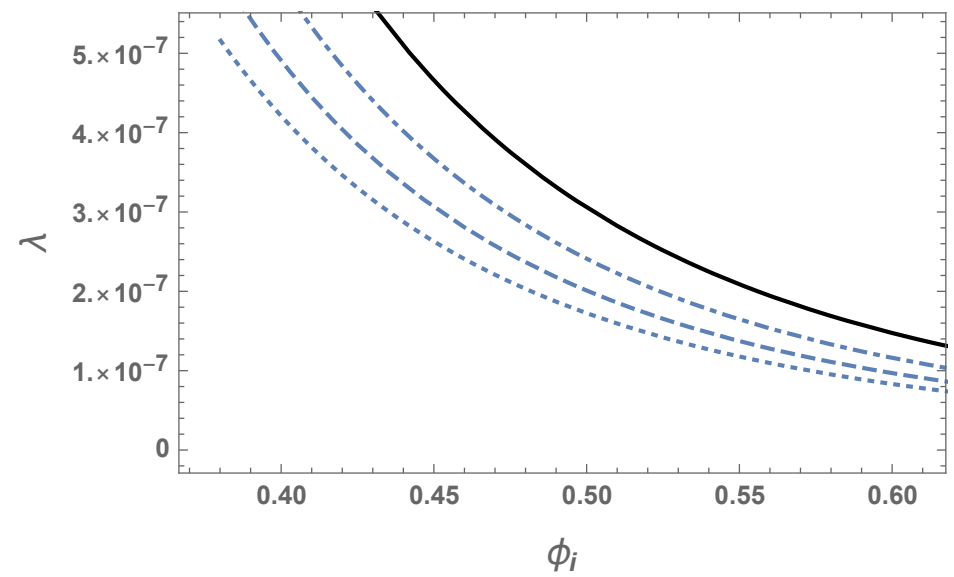

Figure 4: The self coupling $\lambda$ for a large-field $\left(\phi \sim M_{p}\right)$ inflationary scenario corresponding to the left graphic in Fig. (2). Along the shown interval $\lambda$ remains of the order of $10^{-7}$. 
limit case.

Furthermore, it is worth noticing that the contribution of the kinetic term is subdominant in comparison with the potential. This can be easily seen if we take $\beta \sim 10^{15}$, and $N=60$ which after applying the COBE normalization gives $H \sim 3.2 \times 10^{-5} M_{p}$. Taking for instance the case $n=0$ and computing the kinetic coupling term one obtains the following value

$$
9 \beta H^{2} \dot{\phi}_{c}^{2}=9 \beta H^{4}\left(\frac{d \phi_{c}}{d N}\right)^{2} \simeq 2.2 \times 10^{-11} M_{p}^{4}
$$

where $d \phi_{c} / d N$ is obtained by taking the derivative of Eq. 2.15 with respect to the e-foldings number. It becomes clear that the kinetic term is much smaller than the potential, which for the above cases is of order of $V\left(\phi_{c}\right) \sim 10^{-9} M_{p}^{4}$ (after COBE normalization), so that the inflationary period is driven mainly by the Higgs potential. In Fig. 5 we show the theoretical predictions of the model for $\alpha=10^{14}$ embedded in the 2018 Planck constraints including temperature, polarization, and lensing. It is shown the $68 \%$ and $95 \%$ CL regions for the scalar spectral index $n_{s}$ and the tensor to scalar ratio $r$. Colored dots correspond to the theoretical predictions for the models $\beta / \phi^{2}$ (green), $\beta / \phi$ (yellow), $\beta$ (black), and $\beta \phi$ (blue). One can see that for all the cases the theoretical predictions fall inside the 95\% CL region. Moreover, the effect of the non-minimal kinetic coupling is more pronounced in the scalar spectral index than in the tensor to scalar ratio. It is worth noticing that considering $N=50 e$-folds the tensor-to-scalar ratio increments for all cases, while at $N=70$ the increment is on the scalar spectral index side, making in both cases the models less viable.

Analyzing the model in the strong coupling limit we can extract some conclusions regarding the behavior of the observables $n_{s}$ and $r$ for different integer powers $n$. In absence of kinetic coupling all models reduce to the standard canonical field with $\phi^{4}$ potential that gives the know values for $n_{s}$ and $r$ given by

$$
n_{s}=\frac{N-2}{N+1}, \quad r=\frac{16}{N+1},
$$

and at the strong coupling limit $(\beta \rightarrow \infty)$ we find the following values

$$
n_{s}^{(2)}=\frac{2 N-3}{2 N+1}, \quad r^{(2)}=\frac{16}{2 N+1}, \quad n_{s}^{(1)}=\frac{5 N-7}{5 N+2}, \quad r^{(1)}=\frac{32}{5 N+2},
$$




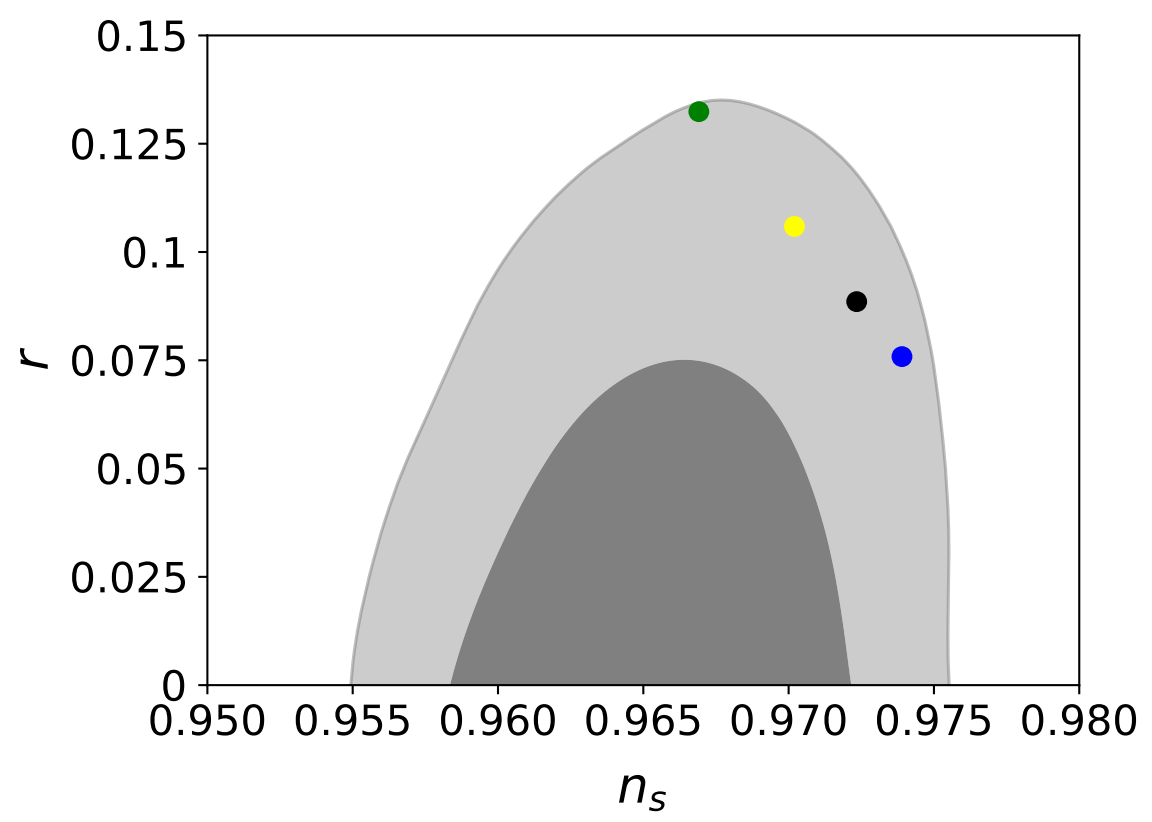

Figure 5: Marginalized 68\% and 95\% CL regions for the scalar spectral index $n_{s}$ and the tensor to scalar ratio $r$ at $k=0.0002 \mathrm{Mpc}^{-1}$. We use the publicly available chains including 2018 Planck data alone, namely, plikHM_TTTEEE_lowl_lowE_lensing. Colored dots show theoretical predictions for the models $\beta / \phi^{2}$ (green), $\beta / \phi$ (yellow), $\beta$ (black), and $\beta \phi$ (blue). These predictions consider $N=60$ and $\alpha \sim 10^{14}$. 


$$
n_{s}^{(0)}=\frac{3 N-4}{3 N+1}, \quad r^{(0)}=\frac{16}{3 N+1}, \quad n_{s}^{(-1)}=\frac{7 N-9}{7 N+2}, \quad r^{(-1)}=\frac{32}{7 N+2},
$$

where the upper labels correspond to the power $n$. These values of $r$ at the strong coupling limit correspond to the minimum value that $r$ can achieve for the corresponding model. Assuming $N=60$ we find that the models with the constant coupling $\beta(n=0)$ and $\beta \phi(n=-1)$ give the lower tensor-to-scalar ratio, $r^{(0)} \approx 0.088$ and $r^{(-1)} \approx 0.076$, that are below 0.1 . For $n=3$ at the strong coupling limit the observables take the values

$$
n_{s}^{(3)}=\frac{3 N-5}{3 N+2}, \quad r^{(3)}=\frac{32}{3 N+2},
$$

which at $N=60$ gives an appropriate value $n_{s} \approx 0.961$ but large tensor-to-scalar ratio $r \approx 0.176$. If we take $n=4$, then $n_{s}$ and $r$ remain the same as in the absence of coupling given by (3.1), which are discarded by the observations, and the only influence of the kinetic coupling is on the values of the scalar field $\phi_{\text {end }}$ and $\phi_{c}$. For $n=5$, the scalar spectral index and the tensor-to-scalar ratio vary between the limits

$$
n_{s}^{(5)}=\frac{N-3}{N+2}, \quad r^{(5)}=\frac{32}{N+2},
$$

making it impossible to realize the slow-roll inflation between the current observed values of $n_{s}$ and $r$. For $n \geq 6$ it can be seen from (2.24) that $\phi_{\text {end }}$ and $\phi_{i}$ can be defined only for values of $\beta$ bellow some finite value that depends on $n$. Thus, taking $n=6$ for instance, the scalar field at the end of inflation becomes $\phi_{\text {end }}=\sqrt{8-\beta / 2}$. This means that the strong coupling limit can not be defined and the slow-roll inflation takes the character of large-field inflation, making it impossible for the self coupling to take values in the interval $\lambda \geq 0.1$. Note also that as $n$ increases the spectral index moves out to the left of the Planck 95\% CL and starting from $n \geq 4, n_{s}$ is located outside that region, while $r$ moves to the top of the 95\% CL region, being located outside the region for $n \geq 3$. In the opposite case, assuming $n<-1$, it is found that in the strong coupling limit the observables tend to the values

$$
n_{s}^{(n<-1)}=\frac{6 N-n(N-1)-8}{N(6-n)+2}, \quad r^{(n<-1)}=\frac{32}{N(6-n)+2} .
$$

This result favor $r$ that decreases as $n$ takes larger negative values, moving towards the $1 \sigma$-region in Fig. 3, but the scalar spectral index increases moving outside the 
$2 \sigma$-region to the right. Thus, for $n<-1$ it is not possible to reach values of $n_{s}$ consistent with the Planck 2018 observational data. In all models considered above the observables $n_{s}$ and $r$ vary between the weak coupling limit, common to all cases, and the strong coupling limit for each case.

\section{Conclusions}

We have presented a study of the slow-roll inflation driven by the Higgs Boson with non-minimal derivative coupling to curvature. The coupling function is of the powerlaw type and we have considered all possible scenarios with positive and negative power. The results depicted in Fig. 1 show that both tensor to scalar ratio $r$ and scalar spectral index $n_{s}$ depend on the parameter $\alpha=\lambda \beta$, where $\lambda$ is the self coupling of the Higgs potential and $\beta$ (the kinetic coupling parameter) is used to set the appropriate values of the scalar field at the horizon crossing. As can be inferred from Fig. 2, for large $\beta$, the rate of decreasing of the scalar field at the horizon crossing slows down as $\beta$ increases, and therefore the observables take practically constant values, very close to the strong coupling limit. The self coupling $\lambda$ is more sensitive to the variation of $\phi_{i}$ (and therefore of $\beta$ ) as shown in Figs. 3 and 4 .

It was found that the only viable values for the power are $n=2,1,0,-1$, which may lead to observables that satisfy the latest Planck constraints on $r$ and $n_{s}$ at the $95 \%$ CL. For large and small values of $\beta$, that give correspondingly small and large values of the inflaton at the horizon crossing, it is possible to satisfy the COBE restrictions with adequate values of the self coupling constant $\lambda$ in a wide interval $\lambda \sim 10^{-11}-0.3$, that covers the Higgs boson phenomenology. Furthermore, the energy contribution of the kinetic term is small enough to be consistent with the slow-roll formalism, driven by the potential in the early stages of the inflation.

Considering all possible powers, it was shown that the models with $n \geq 3$ lead to tensor-to-scalar ratio values $r>0.1$, growing with the increase of $n$, while the scalar spectral index decreases, and starting from $n=4$ is located outside to the left of the 95\% CL region depicted in Fig. 3. The analysis of power-law couplings with $n<-1$ 
shows that while $r$ is favored taking values well below the $r=0.1$ limit, the scalar spectral index increases considerably, moving outside the 95\% CL region to the right. Thus, for $n<-1$ it is not possible to reach values of $n_{s}$ consistent with the Planck 2018 observational data and these models are discarded.

According to the trajectories depicted in Fig. 1, the main flaw of the models is in the tensor-to-scalar ratio, where the only cases in which $r$ takes values below 0.1 are $n=0$ and $n=-1$. One advantage of the models $n=2,1,0,-1$ is that the self coupling $\lambda$ can take values in a wide interval that covers different phenomenological limits. while $n_{s}$ and $r$ (specifically for the cases $n=-1,0$ ) can be consistent with current observations. Particularly interesting is the model with constant coupling $\beta$ $(n=0)$ which was also study quantitatively in [54, 55] where it was also shown that the consistency with the Higgs boson is reached at large coupling regime.

Concerning the reheating process, a preliminary analysis for the present model shows that, when the kinetic coupling is given by an inverse power-law function, the reheating takes place in unusual form. Taking into account the absence of post-inflationary oscillations, the standard reheating mechanisms cannot successfully exit the inflation and reheat the universe. In other words, due to the fact that the coupling becomes very large at the end of inflation, strongly affecting the post-inflationary dynamics, then the simplest reheating process cannot take place. A possible solution to this problem is considering a coupling function of the form $F_{1} \sim\left(\phi+\phi_{0}\right)^{-n}$. Numerical calculations show that this modification allow the appearance of post-inflationary oscillations, leading to a viable reheating. We expect that this little displacement in the field has only an important effect at the end of inflation without substantially affecting the predictions for the power spectra. The study of adequate restrictions for $\phi_{0}$ are being studied.

We have explored the possibilities for the scalar field model, with Higgs potential and derivative coupling to gravity, to achieve successful inflation, where the kinetic coupling function is a monomial of the scalar field. Although we have shown that the models with $n=2,1,0,-1$ are consistent with current observations by the Planck collaboration (falling into the 95\% CL), they might be in trouble when including more data sets such as those by the BICEP2/Keck Array collaboration [75]. A more gen- 
eral kinetic coupling function (e.g., $F(\phi, X))$ or additional curvature corrections could be worked out in order to analyze whether or not a Higgs field with such curvature corrections can satisfy the most stringent constraints.

\section{Acknowledgments}

This work was supported by Universidad del Valle under project CI 71195. WC and DFJ acknowledge financial support from COLCIENCIAS (Colombia).

\section{References}

[1] A. H. Guth and S. H. H. Tye, "Phase Transitions and Magnetic Monopole Production in the Very Early Universe," Phys. Rev. Lett. 44 (Mar, 1980) 631-635. https://link.aps.org/doi/10.1103/PhysRevLett.44.631.

[2] A. D. Linde, "Particle Physics and Inflationary Cosmology," Phys. Today 40 (1987) 61-68.

[3] K. A. Olive, "Inflation," Phys. Rept. 190 (1990) 307-403.

[4] A. R. Liddle and D. H. Lyth, Cosmological inflation and large scale structure. 2000 .

[5] W. H. Kinney, "TASI Lectures on Inflation," arXiv e-prints (Feb, 2009) arXiv:0902.1529, arXiv:0902.1529 [astro-ph.CO].

[6] A. D. Linde, "The Inflationary Universe," Rept. Prog. Phys. 47 (1984) 925-986.

[7] A. A. Starobinsky, "Spectrum of relict gravitational radiation and the early state of the universe," JETP Lett. 30 (1979) 682-685. [,767(1979)].

[8] A. A. Starobinsky, "A New Type of Isotropic Cosmological Models Without Singularity," Phys. Lett. 91B (1980) 99-102. [,771(1980)]. 
[9] A. H. Guth, "Inflationary universe: A possible solution to the horizon and flatness problems," Phys. Rev. D 23 (Jan, 1981) 347-356. https://link.aps.org/doi/10.1103/PhysRevD.23.347.

[10] A. D. Linde, "A New Inflationary Universe Scenario: A Possible Solution of the Horizon, Flatness, Homogeneity, Isotropy and Primordial Monopole Problems," Phys. Lett. 108B (1982) 389-393. [Adv. Ser. Astrophys. Cosmol.3,149(1987)].

[11] A. Albrecht and P. J. Steinhardt, "Cosmology for Grand Unified Theories with Radiatively Induced Symmetry Breaking," Phys. Rev. Lett. 48 (Apr, 1982) 1220-1223. https ://link.aps .org/doi/10.1103/PhysRevLett.48.1220.

[12] A. D. Linde, "Chaotic Inflation," Phys. Lett. 129B (1983) 177-181.

[13] P. J. Steinhardt, N. Turok, and N. Turok, "A Cyclic model of the universe," Science 296 (2002) 1436-1439, arXiv: hep-th/0111030 [hep-th].

[14] R. Brandenberger, "Alternatives to cosmological inflation," Phys. Today 61N3 (2008) 44-49.

[15] A. A. Starobinsky, "Dynamics of Phase Transition in the New Inflationary Universe Scenario and Generation of Perturbations," Phys. Lett. 117B (1982) $175-178$.

[16] V. F. Mukhanov and G. V. Chibisov, "The Vacuum energy and large scale structure of the universe," Sov. Phys. JETP 56 (1982) 258-265. [Zh. Eksp. Teor. Fiz.83,475(1982)].

[17] J. M. Bardeen, P. J. Steinhardt, and M. S. Turner, "Spontaneous creation of almost scale-free density perturbations in an inflationary universe," Phys. Rev. D 28 (Aug, 1983) 679-693. https://link.aps.org/doi/10.1103/PhysRevD.28.679.

[18] R. H. Brandenberger, "Quantum field theory methods and inflationary universe models," Rev. Mod. Phys. 57 (Jan, 1985) 1-60. https://link.aps.org/doi/10.1103/RevModPhys.57.1. 
[19] S. W. Hawking, "The Development of Irregularities in a Single Bubble Inflationary Universe," Phys. Lett. 115B (1982) 295.

[20] A. H. Guth and S.-Y. Pi, "Fluctuations in the New Inflationary Universe," Phys. Rev. Lett. 49 (Oct, 1982) 1110-1113. https://link.aps.org/doi/10.1103/PhysRevLett.49.1110.

[21] F. L. Bezrukov and M. Shaposhnikov, "The Standard Model Higgs boson as the inflaton," Phys. Lett. B659 (2008) 703-706, arXiv:0710.3755 [hep-th].

[22] T. Futamase and K.-i. Maeda, "Chaotic Inflationary Scenario in Models Having Nonminimal Coupling With Curvature," Phys. Rev. D39 (1989) 399-404.

[23] R. Fakir and W. G. Unruh, "Improvement on cosmological chaotic inflation through nonminimal coupling," Phys. Rev. D41 (1990) 1783-1791.

[24] C. Armendariz-Picon, T. Damour, and V. F. Mukhanov, "k - inflation," Phys. Lett. B458 (1999) 209-218, arXiv:hep-th/9904075 [hep-th].

[25] R. Kallosh, A. Linde, and D. Roest, "Superconformal Inflationary $\alpha$-Attractors," JHEP 11 (2013) 198, arXiv:1311.0472 [hep-th].

[26] S. Ferrara, R. Kallosh, A. Linde, and M. Porrati, "Minimal supergravity models of inflation," Phys. Rev. D 88 (Oct, 2013) 085038. https://link.aps.org/doi/10.1103/PhysRevD.88.085038.

[27] S. D. Odintsov and V. K. Oikonomou, "Inflationary Dynamics with a Smooth Slow-Roll to Constant-Roll Era Transition," JCAP 1704 no. 04, (2017) 041, arXiv:1703.02853 [gr-qc].

[28] K. Dimopoulos and C. Owen, "Quintessential Inflation with $\alpha$-attractors," JCAP 1706 no. 06, (2017) 027, arXiv:1703.00305 [gr-qc].

[29] E. Silverstein and D. Tong, "Scalar speed limits and cosmology: Acceleration from D-cceleration," Phys. Rev. D 70 (Nov, 2004) 103505. https://link.aps.org/doi/10.1103/PhysRevD.70.103505. 
[30] M. Alishahiha, E. Silverstein, and D. Tong, "DBI in the sky: Non-Gaussianity from inflation with a speed limit," Phys. Rev. D 70 (Dec, 2004) 123505. https://link.aps.org/doi/10.1103/PhysRevD.70.123505.

[31] X. Chen, "Inflation from warped space," JHEP 08 (2005) 045, arXiv:hep-th/0501184 [hep-th].

[32] D. A. Easson, S. Mukohyama, and B. A. Powell, "Observational signatures of gravitational couplings in DBI inflation," Phys. Rev. D 81 (Jan, 2010) 023512. https://link.aps.org/doi/10.1103/PhysRevD.81.023512.

[33] S. Kachru, R. Kallosh, A. D. Linde, J. M. Maldacena, L. P. McAllister, and S. P. Trivedi, "Towards inflation in string theory," JCAP 0310 (2003) 013 , arXiv:hep-th/0308055 [hep-th].

[34] R. Kallosh, "On inflation in string theory," Lect. Notes Phys. 738 (2008) $119-156$.

[35] D. Baumann and L. McAllister, Inflation and String Theory. Cambridge Monographs on Mathematical Physics. Cambridge University Press, 2015. arXiv:1404.2601 [hep-th]. http://www.cambridge.org/mw/academic/ subjects/physics/theoretical-physics-and-mathematical-physics/ inflation-and-string-theory?format=HB.

[36] L. H. Ford, "Inflation driven by a vector field," Phys. Rev. D 40 (Aug, 1989) 967-972. https://link.aps.org/doi/10.1103/PhysRevD.40.967.

[37] T. Koivisto and D. F. Mota, "Vector Field Models of Inflation and Dark Energy," JCAP 0808 (2008) 021, arXiv:0805.4229 [astro-ph].

[38] A. Golovnev, V. Mukhanov, and V. Vanchurin, "Vector Inflation," JCAP 0806 (2008) 009, arXiv:0802.2068 [astro-ph].

[39] M. Kawasaki, M. Yamaguchi, and T. Yanagida, "Natural Chaotic Inflation in Supergravity," Phys. Rev. Lett. 85 (Oct, 2000) 3572-3575. https://link.aps.org/doi/10.1103/PhysRevLett.85.3572. 
[40] S. C. Davis and M. Postma, "SUGRA chaotic inflation and moduli stabilisation," JCAP 0803 (2008) 015, arXiv:0801.4696 [hep-ph].

[41] R. Kallosh and A. Linde, "New models of chaotic inflation in supergravity," JCAP 1011 (2010) 011, arXiv: 1008.3375 [hep-th].

[42] A. Nicolis, R. Rattazzi, and E. Trincherini, "Galileon as a local modification of gravity," Phys. Rev. D 79 (Mar, 2009) 064036. https://link.aps.org/doi/10.1103/PhysRevD.79.064036.

[43] C. Deffayet, G. Esposito-Farèse, and A. Vikman, "Covariant Galileon," Phys. Rev. D 79 (Apr, 2009) 084003. https://link.aps.org/doi/10.1103/PhysRevD.79.084003.

[44] K. Kamada, T. Kobayashi, M. Yamaguchi, and J. Yokoyama, "Higgs G inflation," Phys. Rev. D 83 (Apr, 2011) 083515. https://link.aps.org/doi/10.1103/PhysRevD.83.083515.

[45] J. Ohashi and S. Tsujikawa, "Potential-driven Galileon inflation," JCAP 1210 (2012) 035, arXiv:1207.4879 [gr-qc].

[46] T. Kobayashi, M. Yamaguchi, and J. Yokoyama, "Inflation Driven by the Galileon Field," Phys. Rev. Lett. 105 (Dec, 2010) 231302. https://link.aps.org/doi/10.1103/PhysRevLett.105.231302.

[47] S. Mizuno and K. Koyama, "Primordial non-Gaussianity from the DBI Galileons," Phys. Rev. D 82 (Nov, 2010) 103518. https://link.aps.org/doi/10.1103/PhysRevD.82.103518.

[48] C. Burrage, C. de Rham, D. Seery, and A. J. Tolley, "Galileon inflation," JCAP 1101 (2011) 014, arXiv:1009.2497 [hep-th].

[49] T. Kobayashi, M. Yamaguchi, and J. Yokoyama, "Generalized G-inflation: Inflation with the most general second-order field equations," Prog. Theor. Phys. 126 (2011) 511-529, arXiv:1105.5723 [hep-th]. 
[50] S. Capozziello, G. Lambiase, and H. J. Schmidt, "Nonminimal derivative couplings and inflation in generalized theories of gravity," Annalen Phys. 9 (2000) 39-48, arXiv:gr-qc/9906051 [gr-qc].

[51] C. Germani and A. Kehagias, "New Model of Inflation with Nonminimal Derivative Coupling of Standard Model Higgs Boson to Gravity," Phys. Rev. Lett. 105 (Jul, 2010) 011302. https://link.aps.org/doi/10.1103/PhysRevLett.105.011302.

[52] L. N. Granda, "Inflation driven by scalar field with non-minimal kinetic coupling with Higgs and quadratic potentials," JCAP 1104 (2011) 016, arXiv:1104.2253 [hep-th].

[53] K. Nozari, M. Shoukrani, and N. Rashidi, "Warm Inflation with Nonminimal Derivative Coupling," Advances in High Energy Physics (04, 2014)

[54] S. Tsujikawa, "Observational tests of inflation with a field derivative coupling to gravity," Phys. Rev. D85 (2012) 083518, arXiv:1201.5926 [astro-ph.C0].

[55] N. Yang, Q. Fei, Q. Gao, and Y. Gong, "Inflationary models with non-minimally derivative coupling," Class. Quant. Grav. 33 no. 20, (2016) 205001, arXiv:1504.05839 [gr-qc].

[56] ATLAS Collaboration, G. Aad et al., "Observation of a new particle in the search for the Standard Model Higgs boson with the ATLAS detector at the LHC," Phys. Lett. B716 (2012) 1-29, arXiv:1207.7214 [hep-ex].

[57] CMS Collaboration, S. Chatrchyan et al., "Observation of a New Boson at a Mass of $125 \mathrm{GeV}$ with the CMS Experiment at the LHC," Phys. Lett. B716 (2012) 30-61, arXiv:1207.7235 [hep-ex].

[58] J. Rubio, "Higgs inflation," Front. Astron. Space Sci. 5 (2019) 50, arXiv:1807.02376 [hep-ph]. 
[59] C. P. Burgess, H. M. Lee, and M. Trott, "Power-counting and the Validity of the Classical Approximation During Inflation," JHEP 09 (2009) 103 arXiv:0902.4465 [hep-ph].

[60] J. L. F. Barbon and J. R. Espinosa, "On the Naturalness of Higgs Inflation," Phys. Rev. D 79 (2009) 081302, arXiv:0903.0355 [hep-ph].

[61] F. Bezrukov, A. Magnin, M. Shaposhnikov, and S. Sibiryakov, "Higgs inflation: consistency and generalisations," JHEP 1101 (2011) 016, arXiv:1008.5157 [hep-ph].

[62] X. Calmet and R. , Casadio, "Self-healing of unitarity in Higgs inflation," Phys. Lett. B 734 (2014) 17, arXiv:1310.7410 [hep-ph].

[63] A. Escriva and C. Germani, "Beyond dimensional analysis: Higgs and new Higgs inflations do not violate unitarity," Phys. Rev. D 95 (2017) 123526, arXiv:1612.06253 [hep-ph].

[64] C. Germani and A. Kehagias, "New Model of Inflation with Nonminimal Derivative Coupling of Standard Model Higgs Boson to Gravity," Phys. Rev. Lett. 105 (2010) 011302, arXiv:1003.2635 [hep-ph].

[65] C. Germani and A. Kehagias, "Cosmological Perturbations in the New Higgs Inflation," JCAP 1005 (2010) 019, arXiv:1003.4285 [astro-ph.CO].

[66] C. Germani, Y. Watanabe, and N. Wintergerst, "Self-unitarization of New Higgs Inflation and compatibility with Planck and BICEP2 data," JCAP 1412 (2014) 009, arXiv:1403.5766 [hep-ph].

[67] R. Metsaev and A. Tseytlin, "Order ?? (two-loop) equivalence of the string equations of motion and the ?-model Weyl invariance conditions: Dependence on the dilaton and the antisymmetric tensor," Nuclear Physics B 293 (1987) 385. 
[68] C. Cartier, J. Hwang, and E. J. Copeland, "Evolution of cosmological perturbations in non-singular string cosmologies," Phys.Rev. D 64 (2001) 103504, arXiv:0106197 [astro-ph].

[69] I. Antoniadis, J. Rizos, and K. Tamvakis, "Singularity-free cosmological solutions of the superstring effective action," Nuclear Physics B 415 (1994) 497, arXiv:9305025 [hep-th].

[70] P. Kanti, J. Rizos, and K. Tamvakis, "Singularity-free cosmological solutions in quadratic gravity," Phys. Rev. D 59 (1999) 083512, arXiv:9806085 [gr-qc].

[71] K. Kamada, T. Kobayashi, M. Yamaguchi, and J. Yokoyama, "Higgs G inflation," Phys.Rev. D 83 (2011) 083515, arXiv:1012.4238 [astro-ph.C0]

[72] J. Ohashi and S. Tsujikawa, "Potential-driven Galileon inflation," JCAP 1210 (2012) 035, arXiv: 1207.4879 [gr-qc].

[73] Particle Data Group Collaboration, M. e. a. Tanabashi, "Review of Particle Physics," Phys. Rev. D 98 (Aug, 2018) 030001. https://link.aps.org/doi/10.1103/PhysRevD.98.030001.

[74] L. N. Granda and D. F. Jimenez, "Slow-Roll Inflation in Scalar-Tensor Models," JCAP 1909 (2019) 007, arXiv: 1905.08349 [gr-qc].

[75] BICEP2, Keck Array Collaboration, P. A. R. Ade et al., "Improved Constraints on Cosmology and Foregrounds from BICEP2 and Keck Array Cosmic Microwave Background Data with Inclusion of $95 \mathrm{GHz}$ Band," Phys. Rev. Lett. 116 (2016) 031302, arXiv:1510.09217 [astro-ph.C0]. 